\title{
High Scalp Tension after Three-Dimensional Titanium Mesh Repair for Skull Defect: 2 Case Reports
}

\author{
Longbiao Xu*, Qichao Chen*, Jingxin Fu, Guosen Du, Yedong Wan, Ming Zhao" \\ Department of Neurosurgery, Zhuji Affiliated Hospital of Shaoxing University, Zhuji People's Hospital of Zhejiang Province, \\ Zhuji, China \\ Email: \#Zhaoming0011@163.com
}

How to cite this paper: $\mathrm{Xu}, \mathrm{L} . \mathrm{B}$., Chen, Q.C., Fu, J.X., Du, G.S., Wan, Y.D. and Zhao, M. (2021) High Scalp Tension after Three-Dimensional Titanium Mesh Repair for Skull Defect: 2 Case Reports. Case Reports in Clinical Medicine, 10, 17-23. https://doi.org/10.4236/crcm.2021.102003

Received: January 13, 2021

Accepted: February 19, 2021

Published: February 22, 2021

Copyright $\odot 2021$ by author(s) and Scientific Research Publishing Inc. This work is licensed under the Creative Commons Attribution International License (CC BY 4.0).

http://creativecommons.org/licenses/by/4.0/

\begin{abstract}
Rationale: The three-dimensional (3D) computer-made titanium mesh is widely used in the skull repair for those patients receiving decompression of the bone flap. It can restore normal anatomy to a greater extent and make a better appearance. Case Presentation: We reported two cases of patients admitted to our hospital who have experienced high scalp tension after skull repair. At first, these two patients underwent decompression of the bone flap, and the physical examination results showed a defect of skull. No neurological symptoms and signs were found. The 3D computed tomography (CT) reconstruction of skull was performed, and then the skull repair with 3D titanium mesh was conducted. But because of high scalp tension, they underwent a second operation, during which we re-trimmed and reduce the arc of the titanium mesh. The scalp incision of both patients healed well and no titanium mesh was exposed. Both patients have a good prognosis. Lessons: We highlight that the high tension of scalp due to overstretching after 3D titanium mesh repair for skull defect should be paid much attention to. Trimming and reducing the arc of titanium mesh is an effective treatment for this situation.
\end{abstract}

\section{Keywords}

High Scalp Tension, Titanium Mesh Repair, Skull Defect

\section{Introduction}

At present, the clinical use of three-dimensional (3D) computer-made titanium ${ }^{\star}$ Longbiao $\mathrm{Xu}$ and Qichao Chen contributed equally.

\#Corresponding author. 
mesh has become the mainstream of skull repair [1], which has the advantages of conforming to the physiological anatomy to a greater extent, fitting closer to the skull, giving ideal appearance and having low infection rate [2]. In spite of some authors believed that complications are rare after cranioplasty with three-dimensional titanium mesh [3], we have noticed that, in our clinical work, after the titanium mesh restored the normal physiological curvature of the margin of skull, the tension of scalp increased due to overstretching, resulting in a series of complications. This is a major drawback that neurosurgeons are not paying much attention to. Besides, as far as we know, there are few case reports about management strategies of high scalp tension after 3D titanium mesh repair for skull defect.

Reviewing 73 patients admitted to our hospital for craniotomy in 2018, there were 2 cases having experienced complications due to high scalp tension after skull repair, and we re-trimmed the titanium mesh in a second operation. After reasonable individualized therapy, both patients achieve satisfactory results with good prognoses. Here the treatment of the two patients is reported as follows, hoped to open a new mind in future clinical cranioplasty.

\section{Ethical Review}

This case report was approved by the clinical ethics committee of the Zhuji Affiliated Hospital of Shaoxing University. The patients had been anonymized and the informed consent was acquired.

\section{Case Report}

\subsection{Case 1}

A female patient who underwent cranial hematoma removal and decompression of the bone flap on the right side following severe craniocerebral trauma due to a traffic accident in May 2018. On July 3, 2018, she was admitted to our department for "skull repair". Her physical examination result showed a defect of $6 \times 8$ $\mathrm{cm}$ on the right side of skull. No neurological symptom and signs were found. Her family, social and past medical histories were noncontributory. No obvious contraindications were found in preoperative laboratory tests. The head computed tomography (CT) was performed before operation (Figure 1(A)). On July 6,2018 , the skull repair was performed under general anesthesia. The material used was 3D computer-shaped titanium mesh based on the result of 3D CT reconstruction. On the second day post-operation, head CT revealed an epidural hematoma under the titanium mesh (Figure 1(B)). The patient was conscious and the vital signs were stable. The bilateral pupils of the patient were symmetrical and sensitive in light reflection. About $150 \mathrm{ml}$ of hemorrhagic fluid was drained from the subcutaneous drainage tube. Subsequently, the changes of consciousness and pupils were closely monitored, and symptomatic treatments such as hemostasis, anti-inflammatory, and brain protection were carried out. On the next day, the patient complained of severe headaches, and obvious 


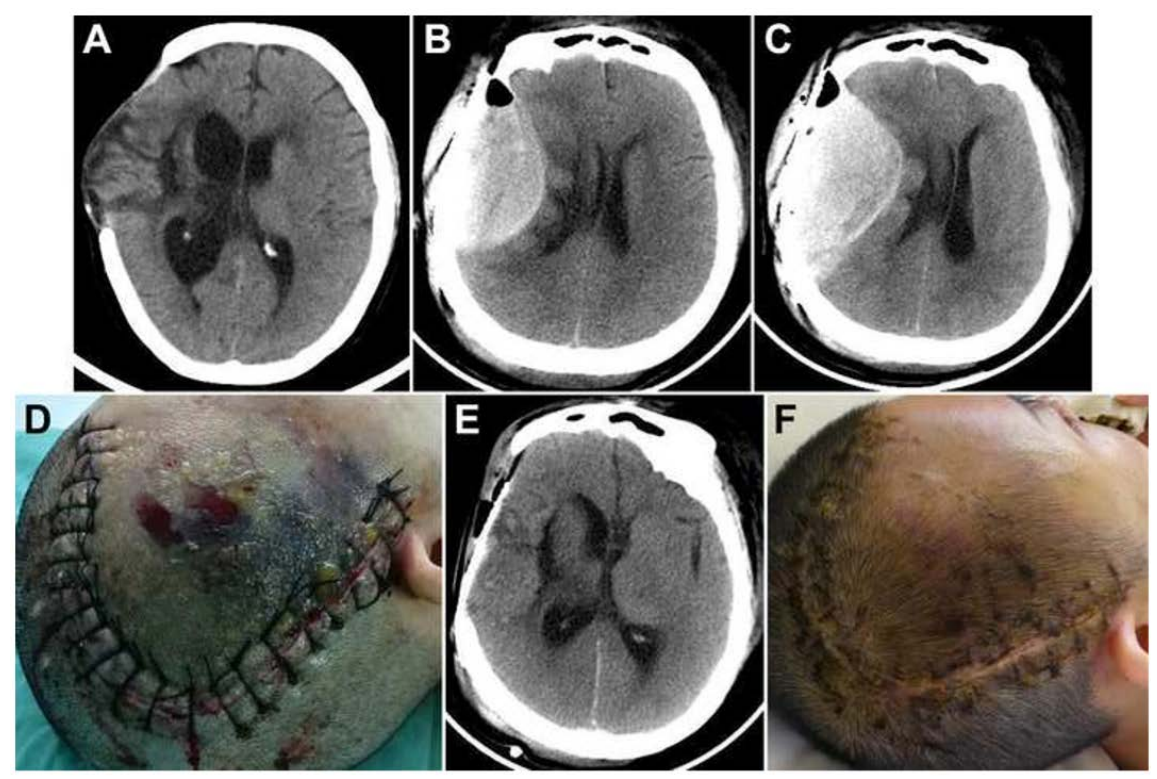

Figure 1. The images of Case 1: The head CT before skull repair showed a defect of $6 \times 8$ $\mathrm{cm}$ on the right side of skull (A); the head CT revealed an epidural hematoma under the titanium mesh after skull repair (B); the head CT showed that the epidural hematoma increased significantly compared with the previous one, and the midline shifted (C); a lot of tension blisters appeared around the incision (D); the result of head CT after the second operation (E); the scalp was well healed (F).

bleeding from the incision. Re-examination of head CT showed that the epidural hematoma increased significantly compared with the previous one, and the midline shifted (Figure $1(C)$ ). At this time, the patient was conscious, and after fully communicating with the patient's family members, they asked for conservative treatment. At 5:00 am on July 9, 2018, the patient's consciousness was significantly lower than before, showing a shallow coma with nausea and vomiting. At the same time, a lot of tension blisters appeared around the incision (Figure $1(D))$. As a result, an emergency operation was performed. During the operation, about $80 \mathrm{ml}$ of right frontotemporal epidural hematoma was removed, and the previous titanium plate was trimmed, part of the titanium mesh and extra screws were discarded. After reshaping, the titanium mesh was fixed to the skull again. The patient was then accessed to neurological intensive care unit for routine treatment (NICU). On July 10, 2018, the patient's consciousness cleared and spontaneous breathing recovered. On July 19, 2018, the result of head CT was satisfactory (Figure 1(E)), and the scalp was well healed (Figure 1(F)). The suture was removed and the patient was discharged on foot. Half a year later, outpatient follow-up showed that the patient was in good condition, which justified our therapeutic direction.

\subsection{Case 2}

A female patient was admitted to our hospital on September 16, 2018 due to "skull defect post-operation due to brain trauma for more than 3 months". Her 
physical examination result showed a skull defect of $8 \times 10 \mathrm{~cm}$ on the right side. Her neurological symptom and signs, as well as her past medical history were negative. No obvious contraindications for surgery. Similarly, the head CT was performed before operation (Figure 2(A)). The skull repair was performed under general anesthesia. The material used was three-dimensional computer-shaped titanium mesh. On the fourth day after surgery, the incision of scalp still showed a lot of exudate (Figure 2(B)). After that, the wound dressing treatment strengthened. On the ninth day, the suture was removed, while the incision was still unhealed and had persistent exudation, the titanium mesh was exposed as well (Figure 2(C)). As a result, a second operation was conducted. After the titanium mesh was fully exposed during the operation, about $3 \times 4 \mathrm{~cm}$ shaped titanium mesh was cut off and discarded, and the corners are trimmed. The remaining titanium mesh was fixed again, and the extra screws were discarded. The result of head CT was also satisfactory (Figure 2(D)). After usual daily therapy, on October 3,2018, the patient was discharged from the hospital on foot with some sutures removed (Figure 2(E)). On April 2, 2019, outpatient follow-up proved the scalp incision healed well with no titanium mesh exposed (Figure 2(F)).

\section{Discussion}

Skull repair after the decompression of the bone flap is of great significance for
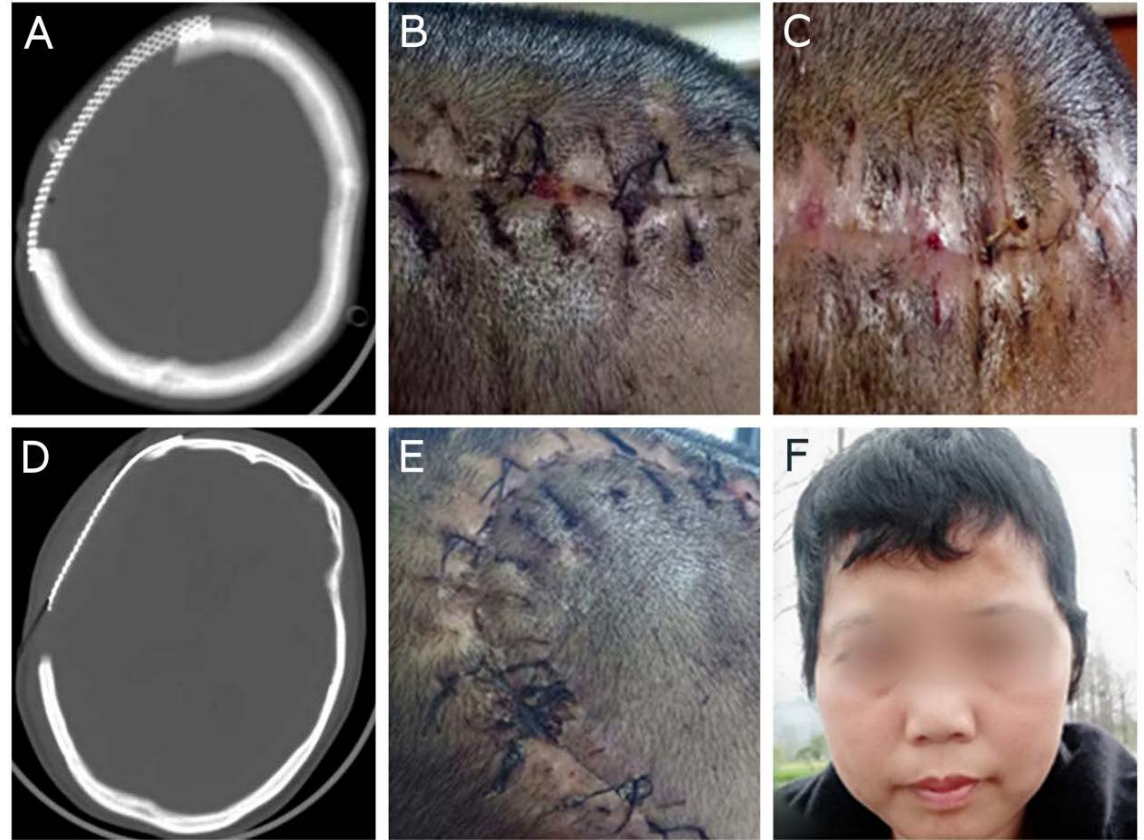

Figure 2. The images of Case 2: The head CT before skull repair showed a defect of $8 \times 10$ $\mathrm{cm}$ on the right side of skull (A); the incision of scalp still showed a lot of exudate (B); after removement of the suture, the incision was still unhealed and had persistent exudation, and the titanium mesh was also exposed (C); the result of head CT after the second operation (D); some sutures were removed (E); the scalp incision healed well with no titanium mesh exposed $(\mathrm{F})$. 
reconstructing the physiological barrier of the cranial cavity, improving cerebral hemodynamics, promoting venous return, and increasing the speed of cerebrospinal fluid circulation [4]. The technology of 3D computer digital forming titanium mesh can restore the integrity of the cranial cavity, reconstruct the physical protective barrier of the cranial contents, relieve the pressure on the cerebral cortex by atmospheric pressure and extra-brain tissue, correct the tortuosity of the cerebral veins in the defect area, and increase the speed of venous return [5] [6] [7].

However, the scalp flap of the patient in case 1 after decompression of the bone flap has been in a low tension state for a long time. Due to the contraction of the scar, the scalp flap area reduces on the bone window. Under the large arc shape of the 3D titanium mesh, although the cranial cavity restores the normal physiological arc to the greatest extent, at this time, the tension of the scalp is too high due to overstretching, which causes the small arteries and veins in the flap to become thinner and even occluded. As a result of the ischemia of the scalp flap, the blood perfusion of the flap is reduced, and the continued ischemia and hypoxia lead to atrophy and thinning of the scalp, resulting in unhealed incision, cracking, thin scalp rupture, and exposed titanium plate [8]. In addition, during the suture of the scalp, because the $3 \mathrm{D}$ titanium mesh needs to be covered by scalp, it is bound to pull the scalp forcibly, resulting in too high tension of the scalp, making the cap fascia on both sides of the scalp not fit well, together with the chronic continued cutting in subcutaneous tissue layers, leading to continuous bleeding under the flap. Besides, scalp flap traction causes thrombus formed during electrocoagulation falling off and bleeding again, especially the related arterial hemorrhage, can cause rapid hematoma formation and severe complications of epidural hematoma [9].

After the 3D titanium mesh is implanted to restore the normal physiological curvature of the skull, excessive scalp tension is a risk factor that cannot be ignored. For some patients with poor flap conditions, especially elderly women, the scalp atrophy, thin scalp, less subcutaneous tissue, poor blood supply to the scalp flap, the high scalp tension are very likely to occur after implantation of the 3D titanium mesh. Mikami et al. reported that owning to the above reasons, elderly women have a high risk of the exposure of titanium mesh after initial surgical site infections [10]. One factor which should be paid attention to is that two patients in our case report are both elderly females with relatively thin scalp. For such patients, the incision design should be done before the first decompression of the bone flap surgery, making the flap appropriately enlarged, so that the margin of the bone windows is in the area of scalp incision. Besides, the bone window should not be enlarged during surgery [11]. The area of skull defect should be fully evaluated before operation, and the cap fascia layer at the scalp incision should be fully released during the operation to avoid pulling forcefully when separating the flap [12]. In addition, we recommend that the surgeon and the patient did not need to pursue a beautiful appearance excessively. Choosing 2D titanium mesh with small arc for implantation and appropriately sacrifice of 
the appearance after repair may reduce the high tension of the scalp flap and ensure the safety of the operation. For those patients who have higher requirements of appearance, scalp dilation before operation or choose new polyetheretherketone (PEEK) materials could be an alternative [9] [13] [14].

\section{Conclusion}

In conclusion, according to our experience, when the scalp tension is too high after 3D titanium mesh repair, which causes complications such as exposed titanium mesh and epidural hematoma, it is not necessary to rush to remove all titanium mesh. Trimming and reducing the arc of titanium mesh may function well, which is recommended.

\section{Authors' Contributions}

Longbiao $\mathrm{Xu}$ and Qichao Chen contributed equally. They, as the main writers of the case report, completed the collection and analysis of relevant documents and wrote the main part of the manuscript. Jingxin Fu, Guosen Du and Yedong Wan took part in the analysis and collation of literature. Zhao Ming is the designer and director of the project, who guided the report writing. All authors read and agree to the final text.

\section{Fund Projects}

This study was funded by the Medical and health technology project of Zhejiang Province (2020RY081), Science and technology bureau project of Shaoxing (2018C30150), Clinical research fund of Zhejiang Medical Association (2019ZYC-A52).

\section{Conflicts of Interest}

The authors declare no conflicts of interest regarding the publication of this paper.

\section{References}

[1] Yang, J., Sun, T., Yuan, Y., Li, X., Yu, H. and Guan, J. (2019) Evaluation of Titanium Mesh Cranioplasty and Polyetheretherketone Cranioplasty: Protocol for a Multicentre, Assessor-Blinded, Randomised Controlled Trial. BMJ Open, 9, e033997. https://doi.org/10.1136/bmjopen-2019-033997

[2] Cabraja, M., Klein, M. and Lehmann, T.N. (2009) Long-Term Results Following Titanium Cranioplasty of Large Skull Defects. Neurosurgical Focus, 26, 7. https://doi.org/10.3171/2009.3.FOCUS091

[3] Chen, S.-T., Chang, C.-J., Su, W.-C., Chang, L.-W., Chu, I.H. and Lin, M.-S. (2015) 3-D Titanium Mesh Reconstruction of Defective Skull after Frontal Craniectomy in Traumatic Brain Injury. Injury-International Journal of the Care of the Injured, 46, 80-85. https://doi.org/10.1016/j.injury.2014.09.019

[4] Mukherjee, S., Thakur, B., Haq, I., Hettige, S. and Martin, A.J. (2014) Complications of Titanium Cranioplasty-A Retrospective Analysis of 174 Patients. Acta Neurochirurgica, 156, 989-998. https://doi.org/10.1007/s00701-014-2024-x 
[5] Roh, H., Kim, J., Kim, J.H., Chong, K., Yoon, W.K., Kwon, T.H., et al. (2019) Analysis of Complications after Cranioplasty with a Customized Three-Dimensional Titanium Mesh Plate. World Neurosurgery, 123, E39-E44.

https://doi.org/10.1016/j.wneu.2018.10.227

[6] Rosinski, C.L., Patel, S., Geever, B., Chiu, R.G., Chaker, A.N., Zakrzewski, J., et al. (2020) A Retrospective Comparative Analysis of Titanium Mesh and Custom Implants for Cranioplasty. Neurosurgery, 86, E15-E22.

https://doi.org/10.1093/neuros/nyz358

[7] Sakamoto, Y. and Arnaud, E. (2016) Multiple Delayed Scalp Reconstruction for Complicated Cranial Defects. Plastic and Reconstructive Surgery-Global Open, 4, e836. https://doi.org/10.1097/GOX.0000000000000862

[8] Zanaty, M., Chalouhi, N., Starke, R.M., Clark, S.W., Bovenzi, C.D., Saigh, M., et al. (2015) Complications Following Cranioplasty: Incidence and Predictors in 348 Cases. Journal of Neurosurgery, 123, 182-188.

https://doi.org/10.3171/2014.9.JNS14405

[9] Lindner, D., Schlothofer-Schumann, K., Kern, B.-C., Marx, O., Muens, A. and Meixensberger, J. (2017) Cranioplasty Using Custom-Made Hydroxyapatite versus Titanium: A Randomized Clinical Trial. Journal of Neurosurgery, 126, 175-183. https://doi.org/10.3171/2015.10.JNS151245

[10] Mikami, T., Miyata, K., Komatsu, K., Yamashita, K., Wanibuchi, M. and Mikuni, N. (2017) Exposure of Titanium Implants after Cranioplasty: A Matter of Long-Term Consequences. Interdisciplinary Neurosurgery, 8, 64-67. https://doi.org/10.1016/j.inat.2017.01.015

[11] Yadla, S., Campbell, P.G., Chitale, R., Maltenfort, M.G., Jabbour, P. and Sharan, A.D. (2011) Effect of Early Surgery, Material, and Method of Flap Preservation on Cranioplasty Infections: A Systemaic Review. Neurosurgery, 68, 1124-1129. https://doi.org/10.1227/NEU.0b013e31820a5470

[12] Lam, S., Kuether, J., Fong, A. and Reid, R. (2015) Cranioplasty for Large-Sized Calvarial Defects in the Pediatric Population: A Review. Craniomaxillofacial Trauma \& Reconstruction, 8, 159-170. https://doi.org/10.1055/s-0034-1395880

[13] Kasprzak, P., Tomaszewski, G., Sujka, W., Kotwica, Z., Stoma, F., Kwinta, B., et al. (2020) Treatment of Very Large Cranial Defects with Individually Shaped Polypropylene Polyester Knitwear Prostheses: A Series of 11 Cases. Journal of Neurosurgical Sciences, 64, 58-65. https://doi.org/10.23736/S0390-5616.16.03149-0

[14] Zhang, Q., Yuan, Y., Li, X., Sun, T., Zhou, Y., Yu, H., et al. (2018) A Large Multicenter Retrospective Research on Embedded Cranioplasty and Covered Cranioplasty. World Neurosurgery, 112, E645-E651.

https://doi.org/10.1016/j.wneu.2018.01.114 